\title{
Zum Auftritt der Figur »Professor« in Berufungsverfahren
}

Julian Hamann, Leibniz Center for Science and Society, University of Hannover

\section{Einleitung: Wissenschaftliche Figuren}

Wissenschaftler*innen verwandeln sich nicht plötzlich in Professor*innen, sobald sie ihren Erstruf annehmen. Selbst das Durchlaufen verschiedener Karrierestufen, die Ansammlung von Leistungsmarkern in Forschung, Lehre und Selbstverwaltung, oder die Sozialisierung in fachlichen Communities sind nur notwendige, aber keine hinreichenden Bedingungen, um Professor*in zu werden. Die Ausgangsannahme meines Beitrags ist, dass es mehr braucht: Um glaubhaft als Professor*in auftreten zu können, müssen Wissenschaftler*innen als professoraler Jemand anerkannt werden. Die Frage danach, wie man Professor*in wird, ist also immer auch eine Frage danach, wie die Figur »Professor « inszeniert und besetzt wird. ${ }^{1}$

Die Figur »Professor « ist eine diskursive Adresse, der bestimmte Erwartungen, Rechte und Pflichten zugeschrieben werden. Analytisch wird damit ein wechselseitiger Prozess zwischen Innen und Außen in den Blick genommen, eine Beziehung zwischen Dispositionen und Praktiken einer Person und den Erwartungen, Rechten und Pflichten, die von außen an diese Person herangetragen werden. ${ }^{2} \mathrm{Um}$ sich als »Professor « glaubhaft in Szene zu setzen, müssen Wissenschaftler*innen die Eigenschaften, die für »Professoren« im Feld als notwendig erachtet werden, ausbilden, und diese relevanten Eigenschaften müssen von ihren Peers anerkannt und attestiert werden - ein Wechselspiel, das nur analytisch sauber zu trennen ist. ${ }^{3}$

\footnotetext{
${ }^{1}$ Die Figur »Professor « ist eine analytische Kategorie, deren grammatikalisches Geschlecht maskulin ist. Im Gegensatz dazu bezeichnet die Schreibweise »Professor*in«Personen, die Figuren inszenieren und Sprecherpositionen bekleiden können.

${ }^{2}$ Foucault, Michel: The Government of Self and Others. Lectures at the Collège de France 1982/83, New York 2010; Alkemeyer, Thomas u.a. (Hg.): Selbst-Bildungen. Soziale und kulturelle Praktiken der Subjektivierung, Bielefeld 2013.

${ }^{3}$ Etzemüller, Thomas: Der »Vf. « als Subjektform. Wie wird man zum »Wissenschaftler« und (wie) lässt sich das beobachten?, in: Alkemeyer, Thomas u.a. (Hg.): Selbst-Bildungen. Soziale und kulturelle Praktiken der
} 
Es wäre jedoch unterkomplex, die Figur »Professor « als eine holistische Subjektposition zu definieren, also anzunehmen, dass Personen irgendwie und irgendwann einfach nur »Professor « sind. Personen, die in der Wissenschaft tätig sind, müssen ständig eine ganze Reihe von Figuren in Szene setzen, die auf sehr unterschiedliche Weise interpretiert werden. In Texten inszenieren Wissenschaftler*innen etwa die Figur »Autor«; sofern sie dies glaubhaft tun, werden sie zitiert und fungieren dann als Referenz für inhaltliche Behauptungen. ${ }^{4}$ Als »Autor des vorliegenden Textes « kann ich meine soeben getätigte Aussage zur Autorschaft über die Technik der Fußnote mit einer Referenz versehen, in welcher der »Autor Michel Foucault « einen Auftritt hat. ${ }^{5}$ Leser*innen dieses Textes erhalten so einen Anhaltspunkt zur intellektuellen Verortung der von mir getätigten Aussage, während der Figur »Autor Michel Foucault« nicht nur eine inhaltliche Aussage zugeschrieben, sondern auch die Anerkennung zuteil wird, für eben jene Aussage eine zentrale Referenz zu sein. ${ }^{6}$ Sprachlich kann die Figur des »Autors « auch mit dem Kürzel »Vf.« markiert werden. ${ }^{7}$ Die Selbstbezeichnung »Vf.« ist eines von vielen sprachlichen Mitteln der Depersonalisierung und Selbstdistanzierung von Autor*innen. Konsequenter als viele Sozial- und Geisteswissenschaften verfahren hier übrigens die meisten Naturwissenschaften, in deren Publikationen alles Subjektive und Individuelle nicht nur distanziert, sondern weitgehend unsichtbar gemacht wird. Sofern die Figur »Autor« überhaupt noch sprachlich sichtbar ist, fungiert sie als neutrales Medium, das von der Natur produzierte und daher objektive Aussagen übermittelt. ${ }^{8}$

Wissenschaftler*in zu sein erschöpft sich aber gerade nicht darin, als »Autor« anerkannt und positioniert zu werden. Bekanntlich sind Wissenschaftler*innen nicht nur körperlose intellektuelle Entitäten, denen man in Texten begegnet. Auf den Bühnen von Institutsfluren oder Besprechungsräumen treten sie etwa auch als »Kollege« auf. Auch diese Figur ist eine Adresse für die Zuschreibung von Erwartungen, Rechten und Pflichten. Je nachdem, wie sie

Subjektivierung, Bielefeld 2013. S. 175-196; Etzemüller, Thomas: Ins »Wahre« rücken. Selbstdarstellung im Wissenschaftsbetrieb, in: Merkur 69, 2015, H. 797, S. 31-43.

${ }^{4}$ Vgl. dazu auch den Beitrag von Lena Vöcklinghaus in diesem Band.

${ }^{5}$ Foucault, Michel: What is an author?, in: Foucault, Michel (Hg.): Language, Counter-memory, Practice: Selected Essays and Interviews, New York, NY 1977. S. 113-138.

${ }^{6}$ Biagioli, Mario/Galison, Peter (Hg.): Scientific Authorship. Credit and Intellectual Property in Science, London 2003.

${ }^{7}$ T. Etzemüller: Der »Vf.« als Subjektform.

${ }^{8}$ Latour, Bruno/Woolgar, Steve: Laboratory Life: The Construction of Scientific Facts, Princeton, NJ 1979; Shapin, Steven/Schaffer, Simon: Leviathan and the Air-Pump. Hobbes, Boyle, and the Experimental Life, Princeton, NJ 2011. 
die Figur »Kollege« inszenieren, können Wissenschaftler*innen kooperativ oder eigenbrötlerisch, verlässlich oder sprunghaft, anderen gegenüber arrogant oder servil sein. Zur Inszenierung des »Kollegen« kann auch gehören, in die Kaffeekasse des Instituts einzuzahlen und an einer Weihnachtsfeier teilzunehmen, also Erwartungen zu entsprechen, die sich eben nicht aus der Rolle des »Autors « ergeben. ${ }^{9}$

Es gibt viele Situationen an denen sich aufzeigen lässt, dass die Trennung solcher Figuren analytischer Art ist. Bereits zwischen den gerade eingeführten Figuren »Autor« und »Kollege « gibt es zahlreiche Überschneidungen. Als »Kollege« lassen Wissenschaftler*innen zufällig einen Ausdruck ihres neuesten Artikels am Institutsdrucker liegen, um auf ihre Produktivität als »Autor« aufmerksam zu machen. Als »Autor « danken Wissenschaftler*innen in der ersten Fußnote einer Publikation oft jenen Personen, die ihnen eben gerade als »Kollege« hilfreiche Rückmeldungen zum Manuskript gegeben haben. Systematisch verschränken sich die Figuren »Kollege« und »Autor« auch auf Konferenzen und Tagungen. Hier treten Wissenschaftler*innen als »Autoren« auf, die ihre Papers zur Diskussion stellen, für inhaltliche Argumente einstehen und Ideen entwickeln. ${ }^{10}$ Verbunden mit diesem intellektuellen Austausch sind aber Begegnungen unter »Kollegen«, die sich von vorherigen Konferenzen kennen oder einander vorgestellt werden, in der Pause über Fußball sprechen, gekonnt netzwerken oder sich schüchtern an ihrer Kaffeetasse festhalten. ${ }^{11}$ Auf Konferenzen müssen sich Wissenschaftler*innen als »Autor « und »Kollege« inszenieren und werden im Falle einer gelungenen Inszenierung von anderen Wissenschaftler*innen entsprechend anerkannt.

Es ließen sich noch viele weitere Figuren nennen. So stellen Wissenschaftler*innen für Studierende »Lehrer« dar, haben Auftritte als »Manager« von Forschungsprojekten oder als »Experte« in einem Zeitungsinterview. Auch hier gilt, was ich für »Autor « und »Kollege« argumentiert habe: Man ist nicht einfach so Lehrer*in, Forschungsmanager*in und Expert*in. Personen müssen in einem sozialen Prozess zu solchen gemacht werden und diese Figuren

\footnotetext{
${ }^{9} \mathrm{Vgl}$. auch den Beitrag von Sabrina Deigert in diesem Band.

${ }^{10}$ Gross, Neil/Fleming, Crystal: Academic Conferences and the Making of Philosophical Knowledge, in: Camic, Charles u.a. (Hg.): Social Knowledge in the Making, Chicago, IL 2011. S. 151-179; Söderqvist,

Thomas/Silverstein, Arthur M.: Participation in Scientific Meetings: A New Prosopographical Approach to the Disciplinary History of Science - The Case of Immunology, 1951-72, in: Social Studies of Science 24, 1994, S. 513-548.

${ }^{11}$ Henderson, Emily F.: Academic conferences: representative and resistant sites for higher education research, in: Higher Education Research \& Development 34, 2015, S. 914-925; Skelton, Alan: Conferences, conferences, conferences?, in: Teaching in Higher Education 2, 1997, S. 69-72.
} 
immer wieder neu aufführen, ausfüllen und behaupten. Die entsprechenden Prozesse fußen wesentlich auf Praktiken der Zuschreibung, Bewertung und Anerkennung.

Ich will in meinem Beitrag die Inszenierung und Besetzung der Figur »Professor « diskutieren. Die Besonderheit dieser Figur ist, dass sie fest in einen organisationalen Kontext eingebettet ist. Mit anderen Worten: Während es für den »Professor « viele Bühnen gibt, vermittelt sich das Drehbuch zu dieser Figur, die an sie gekoppelten Erwartungen, Rechten und Pflichten, zu einem großen Teil über die organisationale Mitgliedschaft in einer Hochschule. Was es heißt, Professor*in zu sein, wie man also einen »Professor « aufzuführen hat, ergibt sich zumindest in Deutschland wesentlich aus Lehrverpflichtungen, dem Promotionsrecht, dem Beamtenstatus mit entsprechender Vergütung, nicht zuletzt auch aus der Leitung eines Lehrstuhls, aus der sich wiederum eine inhaltliche wie auch personelle Leitungsrolle ergibt. Über den organisationalen Kontext hinaus ist die Figur »Professor « auch Adresse für Zuschreibungen eines bestimmten Status im wissenschaftlichen Feld und in der Gesellschaft. Den »Professor « lediglich als eine institutionelle Statusposition zu verstehen, würde demnach zu kurz greifen, weil damit erstens nur ein Bruchteil der Subjektposition sichtbar wird, und weil zweitens vollkommen unklar bleibt, wie diese Position mit ihrem Status, also ihren Rechten, Pflichten und den an sie gekoppelten Erwartungen, eigentlich zustande kommt. Wie also wird die Figur »Professor « inszeniert und besetzt?

\section{Methodologie und Theorie}

Eine zentrale Bühne für die Aufführung der Figur »Professor« sind Berufungsverfahren. Natürlich werden Wissenschaftler*innen auch in anderen Situationen als professoraler Jemand anerkannt. Die Überzeugungskraft der Inszenierung eines »Professors« hängt geradezu davon ab, dass sie sich im alltäglichen Auftritt vollzieht, etwa in der beiläufig richtigen Sprache, Mimik und Gestik oder im angemessenen Gefühl für timing und Präsenz. Demgegenüber sind Berufungsverfahren außeralltäglich. Sie haben dennoch eine besondere Bedeutung für den »Professor«, weil die Figur hier einen wichtigen Auftritt hat. Berufungsverfahren können als ein Komplex von Selbst- und Fremdpräsentation, Bewertung und Beglaubigung verstanden werden. In ihrem Rahmen wird eine (je nach Verfahren spezifische) Version von »Professor« in Szene gesetzt, indem für eine vakante Stelle Erwartungen, Interessen und Ansprüche mobilisiert werden. Kandidat*innen konkurrieren mit 
ihren (weit über das eigentliche Verfahren hinausreichenden) Darstellungen von Professorabilität darum, für die Besetzung der so inszenierten Figur ausgewählt zu werden.

Berufungsverfahren illustrieren, wie sich die Figuren, die ich eben analytisch unterschieden habe, empirisch überschneiden und überlagern: Kandidat*innen werden in Berufungsverfahren mindestens als »Autor « und »Lehrer«, wohl auch als »Kollege« und offenbar zunehmend auch als »Forschungsmanager« bewertet. Die Anerkennung als ein Jemand, der professoral ist, erfolgt auf Grundlage der Einschätzung, dass eine Person eine kooperative Kollegin, eine leider nur mittelmäßige Betreuerin für Doktoranden, aber dafür eine international anerkannte Expertin für neuere Wirtschaftsgeschichte ist. Es sind genau die hinter diesen Zuschreibungen stehenden Bewertungsprozesse, die mich in diesem Beitrag interessieren. Sie entscheiden, ob und wie eine Person als professoraler Jemand anerkannt und für eine Figur »Professor« besetzt wird.

Um mich diesen sozialen Prozessen zu nähern, habe ich Berufungsakten von 145 Berufungsverfahren untersucht, die zwischen 1950 und 1985 an 16 deutschen Universitäten in Germanistik und Geschichtswissenschaft stattgefunden haben. Der gesamte Korpus umfasst etwa 1.500 archivierte Dokumente, von Bewerbungen mit Anschreiben, Curriculum Vitae (CV) und Publikationsliste bis zu Kommissionsprotokollen. Besonders relevant für den vorliegenden Beitrag sind zum einen externe Gutachten, in denen Peers Kandidat*innen aus der Perspektive ihres Forschungsfeldes bewerten, und zum anderen Laudationes, die Kommissionen über Kandidat*innen anfertigen, um die Auswahl für die Berufungsliste zu legitimieren. Dieses empirische Material muss, so wie jedes andere Material auch, methodologisch hinsichtlich seines Entstehungs- und Produktionskontextes reflektiert werden. ${ }^{12}$ Versteht man Berufungsakten historiographisch-positivistisch als Quelle, so geben sie Auskunft über Stellenausschreibungen, den Kreis der Kandidat*innen und Kommissionsmitglieder, hinzugezogene Gutachter*innen, Listenplätze oder die Dauer eines Verfahrens. Aus quellenkritischer Perspektive müssen viele darüber hinausgehende Informationen hinterfragt werden. Was verraten uns Berufungsakten schon darüber, ob Gutachten mit Blick auf bestimmte Kandidat*innen bestellt waren und wie sich die soziale

\footnotetext{
${ }^{12}$ Für eine ausführlichere Entwicklung des folgenden Arguments siehe Hamann, Julian: Berufungsakten als Datensorte für die Geschichte der Soziologie, in: Moebius, Stephan/Ploder, Andrea (Hg.): Handbuch Geschichte der deutschsprachigen Soziologie. Bd. 2: Forschungsdesign, Theorien und Methoden Wiesbaden 2016b. S. 243 255.
} 
Dynamik innerhalb der Kommission situativ entwickelt und zu bestimmten Entscheidungen geführt hat? Zeigen Berufungsakten überhaupt, wie Berufungsverfahren »wirklich« ablaufen?

Alternativ zur historiographisch-positivistischen Lesart können Berufungsakten diskursanalytisch-ethnomethodologisch nicht als Quelle, sondern als Phänomen betrachtet werden. ${ }^{13}$ Dokumente erscheinen dann nicht mehr als quellenkritisch verdächtige Container für Inhalte, sondern als Agenten, die »eigenwillig« in ihrer organisationalen Umwelt wirken und mit ihr interagieren. ${ }^{14}$ Mit dieser Perspektivenverschiebung geraten Bewerbungen, Gutachten, Protokolle und Laudationes nicht aus dem Blick. Es tritt aber die Frage in den Vordergrund, wie diese Dokumente in organisationalen Kontexten produziert und dann verwendet werden, welchen Zweck sie für die Dokumentation des Verfahrens erfüllen, welche Szenen sie im Verlauf eines Verfahrens begleiten und was sie zur Inszenierung eines »Professors« beitragen.

Der diskursanalytisch-ethnomethodologische Zugriff auf das Material erlaubt es, den bürokratischen Vorgang zu untersuchen, in dem eine Figur »Professor« inszeniert und die für ihre Besetzung notwendige Darstellung von Professorabilität durch Kandidat*innen bewertet und beglaubigt wird. Der diskursanalytisch-ethnomethodologische Zugang ist auch deshalb wichtig, weil er auf den spezifischen organisationalen Kontext der Universität verweist, in den ein jeweiliger »Professor« eingebettet ist. Wissenschaftler*innen können, wie erwähnt, nur zu einem professoralen Jemand werden, wenn sie in Berufungsverfahren als professoral anerkannt werden. Dieses bürokratische Verfahren ernst zu nehmen bedeutet, die Performativität von Dokumenten wie CVs, Gutachten und Laudationes im Verfahren zu reflektieren.

Um mich den aufgeworfenen Fragen zu nähern, betrachte ich das Material aus der analytischen Perspektive der Soziologie der Bewertung. Die Bewertungssoziologie ist ein recht heterogenes Feld, das wichtige Erkenntnisse über die Praktiken und Prozesse

\footnotetext{
${ }^{13}$ Die analytische, in der Praxis nicht immer klar zu treffende Unterscheidung zwischen Dokumenten als Quelle und als Phänomen ist angelehnt an Prior, Lindsay: Repositioning Documents in Social Research, in: Sociology 42, 2008, S. 821-836.

${ }^{14}$ Diese Position findet sich in organisationssoziologischer wie in archivwissenschaftlicher Literatur, vgl. Cooren, François: Textual Agency: How Texts Do Things in Organizational Settings, in: Organization 11, 2004, S. 373-393; Trace, Ciaran B.: What is recorded is never simply 'what happened': Record keeping in modern organizational culture, in: Archival Science 2, 2002, S. 137-159.
} 
ermöglicht, mit denen Wertigkeiten festgestellt und institutionalisiert werden. ${ }^{15}$

Bewertungssoziologische Ansätze stellen heuristische Mittel bereit, um jene sozialen Prozesse $\mathrm{zu}$ untersuchen, in denen, wie im vorliegenden Fall, Wertigkeiten und Bedeutungen verhandelt und hergestellt werden. Die Literatur liefert eine Reihe von Beispielen für die Bewertung von Wissenschaftler*innen. ${ }^{16}$ So ist etwa untersucht worden, wie potenzielle Doktorand*innen anhand verschiedener Leistungskonzepte in Empfehlungsschreiben bewertet werden, oder wie Kandidat*innen in Berufungsverfahren von den Zuschreibungslogiken »Exzellenz « und »Gender « zugerichtet werden. ${ }^{17}$ Ich selbst habe anhand von akademischen Nachrufen gezeigt, wie Verstorbene über die Erzählung einer Erfolgsbiographie ein allerletztes Mal bewertet werden. ${ }^{18}$ Im Folgenden schließe ich an die bewertungssoziologische Forschung an und untersuche, wie über Berufungsverfahren verteilte Dokumente Bewertungskriterien mobilisieren, anhand derer eine je nach Verfahren spezifische Version eines »Professors « in Szene gesetzt und die Kandidat*innen mit ihren Darstellungen von Professorabilität bewertet werden. ${ }^{19}$

\section{Die Herstellung der Figur »Professor« und die Bewertung von Professorabilität}

Berufungsverfahren sind Bühnen, auf denen Kandidat*innen mit ihren Bewerbungen virtuell als professorabel inszenieren, bei Probevorträgen reale Auftritte haben und entsprechend bewertet werden. Gleichzeitig werden in jedem Berufungsverfahren Erwartungen und Ansprüche an die zukünftige Inhaber*in einer Professur mobilisiert. Das ist eine Orientierung dafür, wie sich Kandidat*innen aufführen müssen, um eine spezifische Figur des »Professors« zu besetzen. Im Folgenden werde ich untersuchen, anhand welcher Bewertungskriterien ein $»$ Professor« in Szene gesetzt und die Kandidat*innen bewertet werden. Ich werde mich

\footnotetext{
${ }^{15}$ Kjellberg, Hans/Mallard, Alexandre: Valuation Studies? Our Collective Two Cents, in: Valuation Studies 1, 2013, S. 11-30; Lamont, Michèle: Toward a Comparative Sociology of Valuation and Evaluation, in: Annual Review of Sociology 38, 2012, S. 201-221; Peetz, Thorsten u.a. (Hg.): Soziologie der Bewertung. Sonderheft Berliner Journal für Soziologie, Ausgabe 3-4/2016, 2016.

${ }^{16}$ Für einen Überblick siehe Hamann, Julian/Beljean, Stefan: Academic evaluation in higher education, in: Teixeira, Pedro/Shin, Jung Cheol (Hg.): Encyclopedia of International Higher Education Systems and Institutions, Dordrecht 2017. (URL: https://link.springer.com/referenceworken try/10.1007/978-94017-9553-1_295-1 [14.3.2019])

${ }^{17}$ van den Brink, Marieke/Benschop, Yvonne: Gender practices in the construction of academic excellence: Sheep with five legs, in: Organization 19, 2012, S. 507-524; Tsay, Angela u.a.: From character to intellect: changing conceptions of merit in the social sciences and humanities, 1951-1971, in: Poetics 2003, 2003, S. 23 49.

${ }^{18}$ Hamann, Julian: 'Let us salute one of our kind'. How academic obituaries consecrate research biographies, in: Poetics 56, 2016a, S. 1-14.

${ }^{19}$ Für ein ähnliches Argument siehe Hamann, Julian: The making of professors. Assessment and recognition in academic recruitment, in: Social Studies of Science (i.E.).
} 
These are proofs of the final publication available at transcript:

https://doi.org/10.14361/9783839446591-014

nacheinander fachlichen, formalen, standortspezifischen und nicht-akademischen

Bewertungskriterien widmen. ${ }^{20}$

\subsection{Fachliche Kriterien der Bewertung von Professorabilität}

Sehr häufig finden sich im gesamten Material Bewertungen der Kandidat*innen oder ihrer wissenschaftlichen Arbeit aus fachlicher Perspektive. Ein Gutachten hebt etwa hervor, dass ein Kandidat $^{21}$

aus seinem breitgefächerten Wissen durch umsichtige Interpretationen, durch originelle, oft phantasievolle und feinsinnige, aber immer kontrollierte Kombinationen in einer bedeutsamen Problematik neue Wege aufgezeigt [hat]. Deutlich spürbar steht hinter den Untersuchungen kein antiquarisch interessierter Gelehrter, sondern der politisch engagierte Zeitgenosse, der sich die Andersartigkeit von administrativen, politischorganisatorischen Gegenwartsstrukturen an einem historischen Beispiel zu verdeutlichen versucht. ${ }^{22}$

Oft verdichten sich fachliche Zuschreibungen, wie im vorstehenden Zitat deutlich wird, zu Bildern einer Forscher*innenpersönlichkeit; ein Aspekt, auf den noch zurückzukommen sein wird. »Die von der Bewerberin um den Lehrstuhl eingereichten Arbeiten vermitteln das Bild einer geschlossenen wissenschaftlichen Persönlichkeit. Sie verraten sorgsame methodologische Überlegung und strenge Textbeachtung« (Laudatio, 1973).

Fachliche Bewertungen beschränken sich aber nicht auf die Anerkennung von Forschungsleistungen. Kandidat*innen werden nicht nur hinsichtlich ihrer Forschung, sondern auch mit Blick auf ihre Lehre bewertet. Ein Gutachten hält zu einem Kandidaten fest: »Die pädagogischen Fähigkeiten von Herrn Bach, die sich in zahlreichen Seminarübungen und Diskussionen erwiesen haben, bieten die Gewähr, daß er sein Fach nicht nur in der Forschung, sondern auch in der Lehre voll vertreten kann«(Gutachten, 1972). In einem anderen Gutachten heißt es, »dass an der Lehrbefähigung dieses begabten Wissenschaftlers,

\footnotetext{
${ }^{20}$ Es handelt sich hierbei um Kriterien, die eine zentrale Rolle für den Auftritt eine*r Wissenschaftler*in als »Professor « spielen. Zukünftige Forschung zu diesem Thema mag weitere Kriterien zutage fördern, vor allem, wenn sie einen anderen als den aktenmäßigen Zugang zu Berufungsverfahren wählt oder gänzlich andere Bühnen für Inszenierungen der Figur »Professor « untersucht.

${ }^{21}$ Alle Zitate wurden von mir pseudonymisiert, so dass Rückschlüsse auf Namen und Orte unmöglich sind.

${ }^{22}$ Dieses Beispiel zeigt erstens deutlich, dass fachliche Bewertungen oft eine Vorstellung von »guter Forschung « transportieren. Zweitens legt das Beispiel nahe, dass diese Vorstellungen disziplinär gefärbt sind. Auf mögliche disziplinäre Unterschiede kann ich in diesem Beitrag nicht eingehen, stattdessen werden nur Beispiele aus Berufungsverfahren in der Geschichtswissenschaft herangezogen.
} 
der in seiner Forschung selbständige Wege zu gehen vermag, nicht zu zweifeln ist « (1954). Es ist kein Zufall, dass sich in beiden Zitaten zur Eignung in der Lehre Einschübe finden, die noch einmal auf die Forschung verweisen. Hier deutet sich ein Primat der Forschung gegenüber der Lehre an. Dieses Primat zeigt sich bei beiden für die fachliche Bewertung zentralen Dokumententypen, Gutachten und Laudationes, allein anhand der mengenmäßigen Verteilung von forschungs- und lehrbezogenen Bewertungen. Wertzuschreibungen, die die Forschung der Kandidat*innen betreffen, machen in der Regel den Großteil der Gutachten und Laudationes aus. Besonders deutlich zeigt sich der Fokus auf die Forschung dann noch einmal in Gutachten. Hier nehmen Peers aus den Forschungsfeldern der Kandidat*innen die fachliche Bewertung vor. Sie kennen die Kandidat*innen als »Autor« bestimmter Forschung und richten ihre Bewertung entsprechend aus.

Ein wichtiger Modus der fachlichen Bewertung in Berufungsverfahren ist der Vergleich. Es ist auffallend, dass sowohl Kommissionsmitglieder wie auch Gutachter*innen immer wieder in den Modus der vergleichenden Bewertung wechseln. Dabei werden Kandidat*innen in Relation gesetzt zu ihrem Forschungsfeld, zu ihrer Alterskohorte oder zu Mitbewerber*innen im gleichen Verfahren. So heißt es in einem Gutachten: »Als einziger der Kandidaten gehört er zu der im Augenblick modernsten deutschen Historikergruppe, derjenigen der (amerikanisch beeinflussten) >modernen Sozialgeschichte` (mit Wehler, Kocka, Winkler, H. C. Schröder usw.)«(Gutachten 1974). Ein Vergleich der Kandidat*innen untereinander kann entweder eher allgemein bleiben, etwa wenn ein Gutachten über einen Kandidaten festhält: »Unter den drei Bewerbern mit einigermaßen fundierten wirtschaftshistorischen Kenntnissen (Krause, Weber, Schäfer) ist er der stärkste« (Gutachten, 1977). Vergleiche von Kandidat*innen können aber auch sehr konkret spezifische Personen adressieren. Mitunter ist ein konkreter Vergleich ein recht diffiziles Unterfangen. Das zeigt sich in einer Laudatio, in der die Kommission argumentiert, wie sie zu der Auffassung gelangt sei, »daß Frau Dr. Hansen der Vorzug gegenüber Herrn Dr. Frohmann zu geben sei und Herr Dr. Volkers ihm gegenüber nicht zurückgesetzt werden könne, sondern pari passu berücksichtigt werden müsse« (Laudatio 1977).

Die für meinen Beitrag herangezogenen Berufungsakten stammen aus der Geschichtswissenschaft. Typisch für fachliche Bewertungen in dieser Disziplin dürfte sein, dass theoretische Leistungen relativ wenig Beachtung finden und eine oft geradezu handwerklich interpretierte Gründlichkeit und Sorgfalt im Umgang mit Quellen und 
Forschungsliteratur einen hohen Stellenwert hat. Typisch für die Geschichtswissenschaft sind auch intellektuelle Erbschaften, bei denen Berufungen - bis in die 1980er Jahre hinein sogar in der Stellenausschreibung - als »Nachfolge von...« bezeichnet werden. Solche Nachfolgeordnungen werden regelmäßig zu einem expliziten Kriterium der fachlichen Bewertung, etwa wenn es über einen Bewerber heißt: »Es ist kein Zweifel möglich, dass er den Lehrstuhl, auf dem Hermann Fischer und Franz Schmidt wirkten, erfolgreich ausfüllen kann«(Laudatio, 1970). Die für dieses Verfahren spezifische Figur »Professor « ist dann durch vorherige Besetzungen und Inszenierungen gefärbt.

Fachliche Bewertungen durchziehen das gesamte Verfahren. Die Beispielzitate zeigen, dass dabei eine Vielfalt von Kriterien zum Tragen kommt: Oft werden Originalität und Kreativität genannt, nicht selten um zu markieren, wie weit ein*e Kandidat*in den Forschungsstand zu einem bestimmten Thema vorangebracht hat. Ein häufiges Motiv ist auch die Breite der Forschung, wobei dann bewertet wird, inwiefern Kandidat*innen ihren Forschungsgegenstand überblicken und von übergreifenden oder zusammenfassenden Fragestellungen geleitet sind. Spezialistentum steht im Vergleich weniger hoch im Kurs. Schließlich wird immer wieder bewertet, wie klar, fein, oder genau Kandidat*innen ihre Forschung darstellen. Der Vielfalt der auf Forschung bezogenen Kriterien in der fachlichen Bewertung steht eine vergleichsweise unterkomplexe Bewertung der Lehrbefähigung von Kandidat*innen gegenüber. Die relevanten Kriterien beschränken sich hier darauf, wie breit das angebotene Lehrportfolio ist, wie eng es mit der Forschung der Kandidat*innen verwoben ist, und wie viel Erfahrung diese in der Lehre haben.

Der Überblick über die fachliche Bewertung hat illustriert, wie gering standardisiert einige der mobilisierten Kriterien sind. Noch diffiziler werden fachliche Bewertungen, wenn sie, wie gezeigt, vergleichend stattfinden. Wie können so schwer zu greifende fachliche Kriterien vergleichend auf ein sehr heterogenes Feld von Bewerber*innen angewendet werden?

\subsection{Formale Kriterien der Bewertung von Professorabilität}

Formale Bewertungen leisten einen Beitrag zur Reduzierung der Heterogenität des Bewerber*innenfeldes. Sie stellen die formale Eignung aller Kandidat*innen fest und etablieren damit einen einheitlichen Standard, vor dessen Hintergrund fachliche Bewertungen dann eine höhere Aussagekraft haben. Ein wesentliches Kriterium der formalen Eignung von 
Kandidat*innen für eine Professur ist in Deutschland die Habilitation. ${ }^{23}$ Wie wichtig die Habilitation für die Inszenierung von Professorabilität ist, zeigt sich daran, dass sie als formale Anforderung an die Bewerber*innen in vielen Stellenausschreibungen genannt wird. Darüber hinaus wird in allen CVs und in fast allen Gutachten und Laudationes auf Ort und Datum der Habilitation verwiesen. Diese Verweise markieren Kandidat*innen im Laufe des Verfahrens immer wieder mit den entsprechenden formalen Qualifikationen, und damit als potenzielle Darsteller*innen der jeweils inszenierten Figur »Professor «. Nicht habilitierte Kandidat*innen werden in der Regel bereits zu Beginn des Verfahrens aussortiert. Die wenigen Fälle, die es in der Ausnahmesituation der Hochschulexpansion der 1960er und 1970er Jahre trotz fehlender Habilitation in die Begutachtung schaffen, machen den zentralen Stellenwert des formalen Kriteriums Habilitation noch einmal deutlich. Hier wird entweder der Abschluss des Habilitationsverfahrens sehr verbindlich in Aussicht gestellt: »Ende dieses Monats wird die Reinschrift vorliegen, so dass Herr Graf - auch nach Auskunft des Dekans unserer Fakultät - im Sommersemester das Habilitationsverfahren abschließen kann« (Gutachten, 1971). Alternativ wird versucht, äquivalente Leistungen hervorzuheben. So heißt es in einem Fall:

Hinsichtlich der formalen Qualifikationskriterien möchte ich mit Nachdruck unterstreichen, daß die wissenschaftlichen Arbeiten von Frau Hoffmann und Herrn Krüger zweifellos als Leistungen zu gelten haben, die bereits abgeschlossenen Habilitationsschriften anderer Bewerber äquivalent sind. (Gutachten, 1977)

Ein im Vergleich zur Habilitation deutlich seltener und auch weniger offen zutage tretender Gegenstand der formalen Bewertung ist die Erfüllung von Anforderungen, die in der Stellenausschreibung festgehalten sind. Hierbei handelt es sich zwar in der Regel um fachliche Anforderungen, durch die Erwähnung in der Stellenausschreibung werden sie jedoch zu einer Formsache. Im Untersuchungszeitraum von 1950 bis 1985 sind Ausschreibungstexte recht kurz, die Anforderungen sind entsprechend knapp formuliert. Noch 1980 annonciert etwa die Universität Frankfurt:

An der Universität Frankfurt am Main sind unter den Einstellungsvoraussetzungen des $\S 39$ a HUG folgende Professuren zu besetzen: [...] Im Fachbereich Geschichtswissenschaften die Professur (C4) für Alte Geschichte. Aufgabengebiet: Der

\footnotetext{
${ }^{23}$ Zumindest gilt das für den Untersuchungszeitraum. Mittlerweile ist dieses formale Kriterium ergänzt durch alternative Karrierewege, siehe Zimmer, Lena M.: Das Kapital der Juniorprofessur. Einflussfaktoren bei der Berufung von der Junior- auf die Lebenszeitprofessur, Wiesbaden 2018.
} 
Bewerber sollte auch auf dem Gebiet der Verfassungs- und Sozialgeschichte gearbeitet haben. Besondere Qualifikationen: Habilitation ist erwünscht «. ${ }^{24}$ (Hervorh. im Orig.)

Es ist auffallend, dass nur wenige Laudationes auf die in der Ausschreibung festgehaltenen Kriterien Bezug nehmen. Gelegentlich orientieren sich aber Gutachten an diesen formalisierten fachlichen Kriterien. In einem Fall ist die Reihung der Kandidat*innen in einem Gutachten »[i]m Lichte der Kriterien, die im Ausschreibungstext festgehalten worden sind « begründet. In einem anderen Fall heißt es: »Herrn Meyers Qualifikationen habe ich entsprechend dem Ausschreibungstext der Universität Hamburg untersucht «(Gutachten, 1977).

\subsection{Standortspezifische Kriterien der Bewertung von Professorabilität}

Auch wenn die Figur »Professor« an jeder Universität ähnliche Zuschreibungen und Erwartungen mit Blick auf Forschung, Lehre und Verwaltung mit sich bringt, können die Anforderungen an Professor*innen doch von Standort zu Standort und sogar von Professur zu Professur unterschiedlich sein. Entsprechend werden Kandidat*innen auch danach bewertet, ob sie für einen spezifischen Standort professorabel sind. Weil der Untersuchungszeitraum auch die Hochschulexpansion umfasst, sind Belastbarkeit in der Lehre und in administrativen Aufgaben zentrale standortspezifische Kriterien für die glaubhafte Inszenierung von Professorabilität. In einer Laudatio erklärt eine Kommission etwa, sie habe sich bei ihren Listenvorschlägen von der Notwendigkeit leiten lassen, eine Persönlichkeit zu gewinnen,

die nicht nur bedeutende wissenschaftliche Leistungen aufzuweisen hat, sondern auch die Fähigkeit besitzt, die schon vorhandenen Lehrstuhlinhaber in der Bewältigung der unterrichtlichen und organisatorischen Aufgaben des unter Überfüllung leidenden Faches wirksam zu unterstützen. (Laudatio, 1956)

In einer anderen Laudatio heißt es über einen Kandidaten: »Schneider hat sich vielfach in administrativen und organisatorischen Stellungen bewährt, niemand kann den Anforderungen eines grossen Faches an einer grossen Universität besser gewachsen sein« (Laudatio, 1956).

\footnotetext{
${ }^{24}$ Aktuelle Stellenausschreibungen enthalten einen immer komplexeren Kriterienkatalog. Es ist möglich, dass dies mit einem differenzierteren Anforderungsprofil der Figur »Professor « zu tun hat; vgl. dazu Klawitter, Maren: Die Besetzung von Professuren an deutschen Universitäten. Empirische Analysen zum Wandel von Stellenprofilen und zur Bewerber(innen)auswahl, Dissertation 2017; Kleimann, Bernd u.a.: Forschungserfahrung als Berufungskriterium. Anforderungen an zukünftige Professorinnen und Professoren, in: Forschung \& Lehre 2015, 2015, S. 644-645.
} 
Es würde jedoch zu kurz greifen, standortspezifische Bewertungskriterien nur auf lehrbezogene und administrative Fragen zu beziehen. Institute (und auch Universitätsleitungen) versuchen mit Berufungen regelmäßig, ein strategisches Forschungsprofil zu ergänzen. Auf diese Weise werden fachliche Kriterien zum standortspezifischen Faktor: In einer Laudatio stellt die Kommission zu einem Kandidaten fest: »Seine Verbindung von geschichtswissenschaftlichen, wirtschaftswissenschaftlichen und soziologischen Kenntnissen und Fragestellungen paßt in die Ausrichtung dieser Fakultät hinein« (Laudatio, 1979). In einem anderen Fall ist die standortspezifische Bewertung auf fachliche Ausgewogenheit bedacht: »Da der Lehrstuhl für Alte Geschichte wohl auf längere Sicht der einzige der Fakultät bleiben wird, muß Wert darauf gelegt werden, die Stelle mit einem in der römischen und der griechischen Geschichte durch eigene Forschung ausgewiesenen Historiker zu besetzen«(Laudatio, 1974, Hervorh. im Orig.).

Es fällt auf, dass standortspezifische Kriterien oft in Laudationes mobilisiert werden. Zwar können auch Gutachten standortspezifische Kriterien berücksichtigen. Doch Gutachter*innen sind per definitionem extern, ihr Einblick in die Bedürfnisse und Interessen eines Standorts ist daher oft nicht so tiefgehend wie jener der Kommissionsmitglieder. Manche Gutachten entziehen sich unter Berufung auf diese Informationsasymmetrie dem Listenvorschlag. In einem Fall heißt es dazu:

Man kann Ihnen zu dem Niveau der Bewerber nur gratulieren, denn bei allen handelt es sich um gestandene Wissenschaftler, von denen m.E. jeder eindeutig die Qualifikation zur Übernahme eines Lehrstuhls hat. Die Aufstellung der Reihenfolge in der Berufungsliste wird daher sicher auch weitgehend davon abhängen, welche Ausrichtung Sie dem Lehrstuhl zu geben wünschen. (Gutachten, 1974)

In einem anderen Fall platziert ein Gutachter zwei Kandidaten gleichrangig und schließt mit der Feststellung: »[F]ür eine weiterführende Entscheidung könnte etwa die Frage nach der sinnvollen Ergänzung des besetzten Heidelberger althistorischen Lehrstuhls [...] den Ausschlag geben, doch müßte dies eine interne Angelegenheit der betroffenen Fakultät sein « (Gutachten, 1981). Trotz der Informationsasymmetrie zu den Kommissionsmitgliedern setzen Gutachter*innen dennoch regelmäßig dazu an, standortspezifische Kriterien zumindest zu antizipieren. Das kann in sehr allgemeiner Form stattfinden, etwa wenn ein bestimmter Standort für ein spezifisches fachliches Profil bekannt ist. Gutachten halten dann etwa fest, 
»daß sich Herr Neumann vorzüglich in die `Bielefelder Konzeption〈 mit beiderseitigem Gewinn einfügen würde«(Gutachten, 1974). Die gutachterliche Einschätzung standortspezifischer Kriterien findet aber nicht selten auch detaillierter statt:

Als besondere Kriterien kommen für die Universität Gießen hinzu, daß das Spektrum seines Forschungsinteresses wegen der geringen Zahl des Lehrpersonals besonders breit sein muß (mit Schwerpunkt in der späteren Neuzeit) und daß Kontaktmöglichkeiten zu den anderen Fächern des Fachbereichs I sowie zur gesamten Universität bestehen müssen. (Gutachten, 1974)

Auch wenn sich in Gutachten Versuche finden, standortspezifische Aspekte zu antizipieren, ist der hauptsächliche Ort dieses Kriteriums doch eindeutig die Laudatio. Das liegt zum einen daran, dass Laudationes von Kommissionen verfasst werden. Kommissionsmitglieder kennen standortspezifische Interessen und Erfordernisse sehr genau und sind daher in der Lage, diese in die Bewertung einfließen zu lassen. Der zweite Grund für die hervorgehobene Rolle, die standortspezifische Kriterien in Laudationes spielen, liegt in der Funktion, die dieses Dokument im Ablauf von Verfahren spielt. Laudationes begründen die listenplatzierten Kandidat*innen gegenüber den nachfolgenden Entscheidungsinstanzen wie dem Senat oder dem Rektor einer Universität. Es liegt nahe, dass standortspezifische Kriterien für diese Instanzen besonderes Gewicht haben. Kommissionen schreiben die Laudatio strategisch auf diese Leserschaft zu.

\subsection{Nicht-akademische Kriterien der Bewertung von Professorabilität}

Bis hierhin könnte man den Eindruck erhalten, dass die Bewertung von Professorabilität in Berufungsverfahren ausschließlich an akademischen - nämlich fachlichen, formalen und standortspezifischen - Kriterien orientiert ist. Der soziologische Forschungsstand zu wissenschaftlichen Karrieren macht jedoch unmissverständlich klar, dass der legitime Auftritt als »Professor « - also auch die Frage, ob und wie man Professor*in wird - immer auch von nicht-akademischen Faktoren abhängt. ${ }^{25}$ Finden sich nicht-akademische Kriterien der Bewertung auch in Berufungsakten?

\footnotetext{
${ }^{25}$ Siehe nur Beaufaÿs, Sandra u.a. (Hg.): Einfach Spitze? Neue Geschlechterperspektiven auf Karrieren in der Wissenschaft, Frankfurt/M., New York 2012; Zippel, Kathrin: Women in Global Science: Advancing Careers Through International Collaboration, Stanford 2017; Lenger, Alexander: Die Promotion. Ein Reproduktionsmechanismus sozialer Ungleichheit, Konstanz 2008; vgl. auch L.M. Zimmer: Das Kapital der Juniorprofessur.
} 
Zunächst fällt auf, wie regelmäßig Kandidat*innen nicht nur als »Autor «, »Lehrer«, oder »Manager« positioniert, sondern umfassender als Person bewertet werden. Die Bewertung von Professorabilität erstreckt sich, wie übrigens auch ihre Darstellung, häufig auf die gesamte Persönlichkeit. Exemplarisch heißt es in einem Gutachten: »Kohler ist nicht nur ein ausgezeichneter Wissenschaftler, sondern auch eine abgerundete Persönlichkeit und ein hervorragender Charakter von einem stark ausgleichenden Temperament «(Gutachten, 1970). Ein Kandidat empfiehlt sich einem Gutachten zufolge durch Studien, »die nicht nur Beweise seines unermüdlichen Fleißes sind, sondern auch seiner Intensität und seines Historikerblutes « (Gutachten, 1966). Ein anderer Kandidat wird »wegen seines fachlichen Könnens als auch wegen des ganzen Zuschnitts seiner gefestigten Persönlichkeit « als professorabel anerkannt (Gutachten, 1972), einem weiteren Kandidaten wird attestiert, über »die geistig-charakterliche Beschaffenheit und das wissenschaftliche Niveau« zu verfügen, »die für ein Ordinariat erforderlich sind (Gutachten, 1958). Aus sozialer Sicht ist es durchaus nachvollziehbar, dass Kommissionen Wert darauf legen, ihre Entscheidung nicht nur an wissenschaftlichen, sondern auch an zwischenmenschlichen Kriterien zu orientieren. Immerhin wählen Kommissionsmitglieder zukünftige Kolleg*innen aus. Die hier angeführten Beispiele zeigen aber, dass es um mehr geht: Werden holistische, die gesamte Person betreffende Kriterien zur Bewertung von Professorabilität mobilisiert, dann geht es darum, inwiefern Kandidat*innen als Persönlichkeit die Figur »Professor « ausfüllen können. ${ }^{26}$

$\mathrm{Zu}$ den nicht-akademischen Kriterien, anhand derer Darstellungen von Professorabilität bewertet werden, gehören aber nicht nur solche, die charakterliche Merkmale markieren sollen. Bei der Bewertung von Kandidat*innen kommen auch askriptive Merkmale zum Tragen. Viele Kandidat*innen verweisen in ihren CVs explizit auf ihre soziale Herkunft, indem sie die Berufe ihrer Eltern referieren. Kandidat*innen präsentieren sich der Kommission dann etwa als »Sohn des Heilpraktikers Neumann und seiner Frau Else « (CV, 1977) oder als »Sohn des Bankangestellten Richard Lehmann und seiner Ehefrau Maria, geb. Brandt « (CV, 1974). Die zunächst noch häufige Nennung von Elternberufen nimmt allerdings von den 1950ern bis in die 1970er Jahre ab und ist in den 1980er Jahren recht selten geworden. Sehr vereinzelt wird die soziale Herkunft auch über Fremdzuschreibungen in anderen Dokumenten markiert: Laudationes vermerken dann über Kandidaten, er »stammt aus

\footnotetext{
${ }^{26}$ Mit den hier aufscheinenden Mechanismen der wissenschaftlichen Subjektivierung habe ich mich mit Blick auf akademische Nachrufe auseinandergesetzt; vgl. Hamann, Julian: Wie entstehen wissenschaftliche Subjekte? Zum professoralen Ethos akademischer Lebenspraxis, in: Hamann, Julian u.a. (Hg.): Macht in Wissenschaft und Gesellschaft. Diskurs- und feldanalytische Perspektiven, Wiesbaden 2016. S. 83-111.
} 
einem Universitätsjuristenhause « (Laudatio, 1970) oder ist »als Sohn des Arbeits- und Sozialrechtlers Maier in Hamburg geboren« (Laudatio, 1963).

Insgesamt fällt auf, dass die soziale Herkunft der Kandidat*innen wenn überhaupt, dann nur en passant in CVs dokumentiert wird. Bis auf vereinzelte Laudationes werden

Kandidat*innen im restlichen Verfahren nicht mit Blick auf ihre soziale Herkunft positioniert, obwohl den Kommissionen die Informationen durch die CVs vorliegen. Gleiches gilt für das Geschlecht der Kandidat*innen: Auch diese Information liegt den Kommissionen bei allen Kandidat*innen vor, wird aber nie zum expliziten Bewertungskriterium. Das heißt aber nicht, dass Geschlecht für die Aufführung der Figur »Professor« keine Rolle spielt. Die Bedeutung dieses Merkmals zeigt sich allein daran, wie deutlich unterrepräsentiert Frauen unter den Kandidat*innen sind. Unter den 199 Personen, die in den Akten als Kandidat*innen archiviert sind, finden sich sechs Frauen (von denen nur eine berufen wurde). ${ }^{27}$

Berufungsakten vermitteln also den Eindruck, dass soziale Herkunft und Geschlecht bei der Bewertung von Kandidat*innen keine Rolle spielen - obwohl der Kommission die Informationen dazu vorliegen. Dieser Befund ist sicher auch damit begründet, dass es sich bei Berufungsakten um spezifisches Material handelt, das die mit Sicherheit stattfindende Bewertung von Kandidat*innen entlang sozialstruktureller Merkmale zensiert. Aktenkundig wird nur eine offizielle und geglättete Version von Berufungsverfahren, eine Vorderbühne, die zwar nicht genau das widerspiegelt, was sich in Konferenzräumen abspielt, aber dafür relativ präzise vermittelt, welche Kriterien offiziell sagbar und legitim sind. Während Gutachten und Laudationes ein Desinteresse an sozialstrukturellen Merkmalen wie Geschlecht und sozialer Herkunft demonstrieren, ist es bemerkenswert, dass diese Zensur durch das Material nicht prinzipiell alle nicht-akademischen Kriterien unsichtbar macht: Die Bewertung von Kandidat*innen anhand ihrer gesamten Persönlichkeit und als ganze Person ist demnach durchaus sagbar und legitim.

\section{Diskussion: Zum Auftritt der Figur »Professor«}

\footnotetext{
${ }^{27}$ Die Zahl der Kandidat*innen ist in Relation zur Anzahl der untersuchten Verfahren deshalb so niedrig, weil Personen einfach gezählt wurden, selbst wenn sie an mehreren Verfahren als Kandidat*innen beteiligt waren. Die Angaben gelten nur für die 89 in der Geschichtswissenschaft untersuchten Verfahren. In der Germanistik ist die Lage aber nur unwesentlich besser, hier finden sich in 56 Verfahren unter 128 Kandidat*innen zehn Frauen (davon wurden vier berufen).
} 
Für eine glaubhafte Inszenierung müssen Wissenschaftler*innen sich immer wieder aufs Neue als »Professor « aufführen. Dieser soziale Prozess baut auf situierten Praktiken auf und ist niemals abgeschlossen. Berufungsverfahren sind demzufolge weder der erste noch der letzte Akt bei der Anerkennung von Wissenschaftler*innen als professorabel. Immer dann, wenn eine Person als Professor*in handelt und anerkannt wird - beispielsweise von Studierenden, von Kolleg*innen oder in Zeitungsinterviews - wird die Figur »Professor « in Szene gesetzt. Diese Situationen sind über den wissenschaftlichen Alltag verstreut.

Anstatt diesen Situationen weiter nachzugehen, will ich zu meiner Eingangsfrage zurückkehren: Wie wird die Figur »Professor « inszeniert und besetzt, und wie werden in diesem Zuge Wissenschaftler*innen zu einem professoralen Jemand? Mein Beitrag hat deutlich gemacht, dass an diesem Prozess erstens verschiedene Entscheidungsinstanzen beteiligt sind. Ich habe mich vor allem auf externe Gutachter*innen und die Berufungskommission konzentriert sowie auf die von diesen Instanzen produzierten Dokumente, anhand derer Kandidat*innen im Verfahren bewertet werden. Zweitens ist deutlich geworden, dass in den Dokumenten eine Reihe von Bewertungskriterien mobilisiert werden. Anhand von fachlichen, formalen, standortspezifischen und nicht-akademischen Kriterien wird verhandelt, wie glaubhaft Kandidat*innen in ihrer Laufbahn und bei ihren Auftritten im Verfahren selbst Professorabilität darstellen. In der Regel leiten diese Kriterien die Entscheidung an, welche*r Kandidat*in für die Besetzung des jeweils im Verfahren inszenierten »Professors « ausgewählt wird. Der auf diese Weise zu einem professoralen Jemand gemachten Person werden dann professorale Rechte, Pflichten und Erwartungen zuteil, wobei die Person immer wieder dann als »Professor« auftritt, wenn sie sich als Professor*in aufführt oder in Szene gesetzt wird, also entsprechend handelt oder adressiert wird. Es ist wichtig zu erinnern, dass mit der Anerkennung von Professorabilität nicht nur die symbolische Beglaubigung einer wissenschaftlichen Person einhergeht, sondern auch Möglichkeiten der Akkumulation von Ressourcen und Macht geschaffen werden - die Figur des »Professors « markiert etwa in Deutschland auch die Hoheit über Personalentscheidungen, eine intellektuelle Führungsposition und einen Beamtenstatus mit entsprechendem Gehalt.

Mein Beitrag trägt dazu bei, »Professor« als Figur, mithin als Subjektposition, und nicht lediglich als Statusposition in einer organisationalen Hierarchie zu begreifen. ${ }^{28}$ Als wichtige

\footnotetext{
${ }^{28}$ Siehe mit ähnlicher Stoßrichtung: Lamont, Michèle: How to Become a Dominant French Philosopher: The Case of Jacques Derrida, in: The American Journal of Sociology 93, 1987, S. 584-622; Beaufaÿs, Sandra: Wie
} 
Bühne für die Inszenierung und Besetzung dieser Figur sind Berufungsverfahren zumindest in der Soziologie bislang vernachlässigt worden. Abgesehen von einzelnen Studien, die wichtige Einblicke in Berufungsverfahren geben, ${ }^{29}$ ist das Wissen darüber, was ein*e Professor*in eigentlich ist und wie man dazu wird, begrenzt. Mein Beitrag setzt dazu an, einen Teil dieser Forschungslücke zu schließen. Anhand der (1) fachlichen, (2) formalen, (3) standortspezifischen und (4) nicht-akademischen Bewertungskriterien, die für die Inszenierung und Besetzung der Figur »Professor« zentral sind, lassen sich einige ihrer wesentlichen Merkmale skizzieren.

(1) Mit Blick auf die fachliche Bewertung von Professorabilität hat sich gezeigt, dass fachliche Bewertungen häufiger auf Forschung als auf Lehre rekurrieren und dabei auch einen vielfältigeren, differenzierteren Katalog an Kriterien mobilisieren. Für die Inszenierung eines »Professors « heißt das, dass ein Auftritt der Kandidat*innen als »Forscher« offensichtlich einen höheren Stellenwert hat als ihre Selbstdarstellung als »Lehrer«. Der Figur des »Professors « werden demnach bevorzugt forschungsbezogene Rechte, Pflichten und Erwartungen zugeschrieben. Ein »Professor « ist immer schon in fachlich-disziplinäre Räume eingelassen. Das legen auch jene Bewertungskriterien nahe, die disziplinenspezifischer Natur sind oder zumindest eine solche Gewichtung haben. Das Primat der Forschung ist zumindest insofern überraschend, als dass die in der Kommission vertretenen zukünftigen Kolleg*innen als auch die beteiligten inneruniversitären Entscheidungsinstanzen ein ausgeprägtes Interesse daran haben dürften, qualifiziertes, ambitioniertes Lehrpersonal zu rekrutieren.

(2) So wie die fachlichen Bewertungskriterien zeigen, dass ein »Professor« in fachlichdisziplinäre Räume eingelassen ist, machen die formalen Kriterien den organisationalen Kontext der Figur sichtbar. Der organisationale Kontext äußert sich zum einen darin, dass diese Figur »Professor« (auch, aber nicht ausschließlich) in einem bürokratisch reglementierten Verfahren inszeniert und besetzt wird. Dieses Verfahren prüft auch formale

werden Wissenschaftler gemacht? Beobachtungen zur wechselseitigen Konstitution von Geschlecht und Wissenschaft, Bielefeld 2003; Engler, Steffani: »In Einsamkeit und Freiheit «? Zur Konstruktion der wissenschaftlichen Persönlichkeit auf dem Weg zur Professur, Konstanz 2001; Angermuller, Johannes: How to become an academic philosopher. Academic discourse as multileveled positioning practice, in: Sociología histórica 2, 2013, S. 263-289; Angermuller, Johannes: Academic careers and the valuation of academics. A discursive perspective on status categories and academic salaries in France as compared to the U.S., Germany and Great Britain, in: Higher Education 73, 2017, S. 963-980.

${ }^{29}$ Musselin, Christine: The market for academics, New York, NY 2010; M. van den Brink/Benschop, Y.: Gender practices in the construction of academic excellence; Hamann, Julian/Beljean, Stefan: Career gatekeeping in cultural fields, in: American Journal of Cultural Sociology (i.E.). 
Qualifikationen, die Kandidat*innen für die glaubhafte Darstellung eines »Professors « erfüllen müssen. Zum anderen machen die formalen Kriterien deutlich, dass die Figur die Mitgliedschaft in einer Organisation markiert. Für die Mitgliedschaft müssen bestimmte formale Kriterien erfüllt sein. Mit der Mitgliedschaft sind aber auch bestimmte organisationalen Rechte und Pflichten verbunden, die vom Beamtengehalt über die Forschungsfreiheit bis zur Lehrverpflichtung reichen.

In der organisationalen Einbettung und den damit einhergehenden bürokratisch formalisierten Zuschreibungen von Rechten, Pflichten und Erwartungen liegt eine weitere Besonderheit der Figur »Professor « und einer der Hauptunterschiede zu anderen Figuren in der Wissenschaft: $»$ Kollege « etwa ist nicht im engeren Sinne an eine organisationale Mitgliedschaft gebunden. Auch die Figur »Autor« ist nicht bürokratisch formalisiert: Was »Autor«-Sein genau heißt, wie also die jeweilige Figur ausgefüllt und interpretiert wird, variiert zwischen verschiedenen »Autoren $\ll .{ }^{30}$ Erst diese Varianz anderer wissenschaftlicher Figuren macht deutlich, wie formalisiert die Figur des »Professors« ist. Unabhängig von den natürlich sehr unterschiedlichen Arten, das »Wissenschaftler «-Sein zu leben, sind die an das »Professor«Sein geknüpften Rechte, Pflichte und Erwartungen doch erstaunlich stabil. ${ }^{31}$

(3) Im Vergleich zu formalen Kriterien machen standortspezifische Kriterien der Bewertung von Professorabilität deutlich, dass das Drehbuch für die Figur »Professor « zwar über Universitäten und Fächer hinweg relativ stabil ist, aber dennoch an einem spezifischen Standort aufgeführt und interpretiert werden muss. Personen können in Berufungsverfahren immer nur mit Blick auf einen bestimmten Standort zu einem professoralen Jemand werden. Trotz ihrer formalen Stabilität gibt es also keinen »Professor «, der nicht an einer bestimmten Universität und einem konkreten Institut angesiedelt ist und in genau diesem Kontext in Szene gesetzt werden müsste. So formalisiert professorale Rechte und Pflichten auch sein mögen, der praktische Vollzug des »Professor«-Seins findet immer auch vor dem Hintergrund von Erwartungen und Möglichkeiten statt, die sich aus standortspezifischen Bedingungen ergeben.

\footnotetext{
${ }^{30}$ Wofür genau etwa der »Autor Jacques Derrida «steht, hängt stark von den Bedingungen und Anschlüssen auf den kulturellen Märkten ab, auf denen er rezipiert wird. Siehe dazu M. Lamont: How to Become a Dominant French Philosopher; vgl. auch Gross, Neil: Richard Rorty: The Making of an American Philosopher, Chicago 2008.

${ }^{31}$ Das gilt zum einen nur innerhalb nationaler Hochschulsysteme: Während ein »Professor« in Deutschland über alle Fächer und Universitäten hinweg sehr ähnlich definiert ist, gibt es zwischen dem deutschen und dem USamerikanischen »Professor « erhebliche Unterschiede. Zum anderen hat die Novelle des Bundesbesoldungsgesetzes 2002 einen vergleichsweise großen Verhandlungsspielraum geöffnet, mit dem es nun auch in Deutschland stärker variiert, was es heißt, »Professor « zu sein.
} 
(4) Schließlich verweisen nicht-akademische Bewertungskriterien darauf, wie weitreichend die mit der Figur des »Professors« verbundenen Erwartungen sind. Erstens wird bei der Bewertung von Professorabilität dezidiert auch auf charakterliche Professorabilität abgestellt. Man ist »Professor«. Die mit dieser Positionierung verbundenen Bewertungskriterien verweisen darauf, dass die Sozialisierung in das Feld vollumfassend ist. Nicht zufällig werden Professor*innen in Deutschland berufen. Es handelt sich bei einem »Professor « um eine Figur, die man mit seinem ganzen Leben ausfüllt und der man sich als Wissenschaftler*in mit seiner gesamten Persönlichkeit verschreibt. ${ }^{32}$

Zweitens machen nicht-akademische Bewertungskriterien darauf aufmerksam, dass ungleichheitsrelevante Prozesse der Bewertung und Anerkennung nicht nur in die Besetzung institutioneller Statuspositionen eingebaut sind, sondern auch in die Inszenierung eines »Professors «. Die Bewertung von Professorabilität ist - fast demonstrativ - nicht an sozialstrukturellen Merkmalen wie der sozialen Herkunft und dem Geschlecht der Kandidat*innen interessiert. Das zeigt, wie Berufungsverfahren selbst noch in Aktenform als Arenen symbolischer Herrschaft fungieren. Die Figur »Professor « wird in Szene gesetzt, als befinde sie sich in einem sozialstrukturellen Vakuum, ihre Verankerung im sozialen Raum wird verkannt, obwohl diese sozialräumliche Verankerung nicht nur bestens erforscht, sondern auch habituell offensichtlich ist. Dennoch kann die Verkennung der sozialstrukturellen Fundierung des »Professors« nicht überraschen. Wissenschaftler*innen haben die von Pierre Bourdieu als epistemische Doxa beschriebene Tendenz, die sozialen und ökonomischen Voraussetzungen ihrer scholastischen Haltung nicht mitzudenken. ${ }^{33}$ Diese Neigung zeigt sich in Berufungsakten deutlich.

\footnotetext{
${ }^{32}$ Vgl. Beaufaÿs, Sandra: Die Freiheit arbeiten zu dürfen. Akademische Laufbahn und legitime Lebenspraxis in: Beiträge zur Hochschulforschung 37, 2015, S. 40-59.

${ }^{33}$ Bourdieu, Pierre: Praktische Vernunft. Zur Theorie des Handelns, Frankfurt/M. 1998. Siehe dazu auch Etzemüller, Thomas: How to make a historian. Problems in writing biographies of historians, in: Storia della Storiografia 2008, 2008, S. 46-57.
} 